\title{
Perineural Invasion As the Sole Pathologic Risk Factor After Surgical Resection for Head and Neck Squamous Cell Carcinoma
}

\author{
Ryan T. Hughes ${ }^{1}$, Joshua Farris ${ }^{1}$, Cole Steber ${ }^{1}$, Bart A. Frizzell ${ }^{1}$, Kathryn M. Greven ${ }^{1}$ \\ 1. Radiation Oncology, Wake Forest School of Medicine, Winston-Salem, USA
}

Corresponding author: Ryan T. Hughes, ryhughes@wakehealth.edu

\begin{abstract}
Introduction

Postoperative radiotherapy (PORT) is routinely recommended for patients with head and neck squamous cell carcinoma (HNSCC) based on pathologic risk factors (pRFs) such as perineural invasion (PNI). Patients with PNI as the sole pRF after resection of HNSCC are uncommon and their prognosis is less clear. The aim of this study is to assess the role of PNI as a sole risk factor in patients with otherwise pathologically lowrisk HNSCC.
\end{abstract}

\section{Methods}

Patients with HNSCC of the oral cavity, pharynx, or larynx treated with primary surgical resection from 2013 to 2018 were identified from an institutional cancer registry. Those with pRFs (pathologic T3-4 disease, lymphovascular space invasion [LVSI], multiple positive lymph nodes, close [within $2 \mathrm{~mm}$ ] or positive margins, extranodal extension [ENE], or recurrent disease) were excluded, yielding an otherwise pathologically low-risk cohort with or without incidental, pathologic PNI. Locoregional control (LRC), overall survival (OS) and disease-specific survival (DSS) were estimated and compared between PNI groups and by adjuvant therapy.

\section{Results}

A total of 1,058 patients were identified as having undergone surgical resection. Exclusion of patients with other pRFs, those with unknown PNI, and oral cavity patients with depth of invasion $>10 \mathrm{~mm}$ yielded a study cohort of 85 patients. Eight patients (10\% of study group, $<1 \%$ of all patients) had PNI as the sole pRF, none of which had clinical signs or symptoms of perineural tumor spread. The remaining 77 were negative for PNI and thus pathologically low risk. Patients with PNI were more likely to have oral cavity cancer, to be younger, and to have received PORT than those without PNI; no patient received concurrent chemotherapy. At a median follow-up of 46.4 months, two- and five-year LRC rates were $81.4 \%$ and $78.5 \%$, respectively. No differences were noted between PNI-positive and PNI-negative groups $(\mathrm{p}=0.73$ ) or PORT v. no-PORT groups $(\mathrm{p}=0.39)$. While the utility of PORT is not possible to assess given limited sample size, four patients with PNI who did not receive PORT did not experience locoregional failure. Seventeen patients overall experienced locoregional failure and 14 were ultimately salvaged. Five-year OS and DSS were $77.4 \%$ and $90.8 \%$, respectively.

\section{Conclusion}

Patients with pathologically low-risk HNSCC after surgical resection experience high rates of LRC. In this large institutional cohort, PNI as the sole pRF was exceedingly rare, and the benefit of adjuvant therapies is difficult to assess. Within this limitation, PORT remains the standard of care for patients with PNI to reduce the risk of locoregional failure. Further collaborative studies are required to adequately assess the prognostic impact of PNI alone in resected HNSCC.

Categories: Otolaryngology, Radiation Oncology, Oncology

Keywords: perineural spread, perineural invasion, head and neck neoplasms, squamous cell carcinoma

\section{Introduction}

Perineural invasion (PNI), defined as tumor cell invasion into, around, or through nerves, has long been considered a pathologic risk factor (pRF) for recurrence after resection of head and neck squamous cell carcinoma (HNSCC) [1-7]. PNI is often identified in association with other high-risk pathologic features including lymphovascular space invasion (LVSI) and lymph node metastases and as a result has been considered a high-risk factor for local disease recurrence [3,7,8]. Early studies of postoperative radiotherapy (PORT) utilized risk stratification systems that included PNI to guide the use of PORT $[9,10]$. Favorable longterm outcomes after risk-adapted adjuvant radiotherapy, particularly for patients with low-risk pathologic features such as PNI alone [11]. Additionally, currently ongoing clinical trials utilize PNI as an 
indication for PORT (NCT00956007, NCT01898494, NCT02215265). Few studies have isolated the risk factor in the context of otherwise low-risk pathologic features to assess its individual impact on locoregional disease control. As a result, limited data exist regarding the prognostic role of PNI as the sole pRF [12]. In this study, we aimed to assess the frequency of PNI as the sole pRF as well as the prognostic impact of PNI on a cohort of otherwise pathologically low-risk patients after surgical resection for HNSCC.

\section{Materials And Methods}

In this Institutional Review Board-approved retrospective cohort study, all patients treated consecutively with surgical resection for HNSCC of the oral cavity, pharynx, or larynx between 2013 and 2018 were identified from an institutional cancer registry. Patients with pRFs other than PNI were excluded. These risk factors included pT3-4 (American Joint Committee on Cancer 7th Edition) disease, LVSI, close (within 2 mm) or positive margins, multiple positive lymph nodes, extranodal extension (ENE), or recurrent disease were excluded. Patients with oral cavity primary tumors with extensive depth of invasion (DOI) defined as greater than $10 \mathrm{~mm}$ were also excluded, as DOI is an independent risk factor in these patients [13]. Those with unknown or not reported PNI status after a review of the pathology report were excluded.

Locoregional recurrence was defined as clinical or pathological evidence of disease at the primary tumor site or neck. Locoregional control (LRC) was defined as the time from surgery to locoregional recurrence or last follow-up (right censor). Overall survival (OS) was defined as the duration from surgery to death from any cause or last follow-up. Disease-specific survival (DSS) was defined as the time from surgery to death due to the same disease.

Descriptive analyses were performed and compared between groups using the chi-square and Fisher's exact test as appropriate for categorical variables and the Mann-Whitney U test for continuous variables. Time-toevent outcomes were estimated using the Kaplan-Meier method and compared across strata using the logrank test. Statistical analyses were performed using R version 3.6 (R Foundation for Statistical Computing, Vienna, Austria).

\section{Results}

A total of 1,058 patients were treated with upfront surgical therapy. Excluding patients with other high-risk pathologic features (pT3-4 disease, LVSI, close or positive margins, multiple lymph nodes or ENE) or recurrent disease yielded a group of 100 patients. Seven patients did not have PNI reported in the pathology report and were categorized as unknown and excluded, as were eight patients with high-risk oral cavity cancers with DOI $>10 \mathrm{~mm}$. The remaining 85 patients ( $8.0 \%$ of all identified patients) formed the study cohort. Patient baseline characteristics are described in Table 1. Overall, eight patients (10.4\% of the study cohort, $0.8 \%$ of all patients treated within this timeframe) had incidental PNI as the sole pRF; 77 patients were negative for PNI and thus pathologically low risk. There were no differences in gender, smoking status, HPV status, pathologic T or N stage between groups that did or did not have PNI as the sole pRF. Patients with PNI had oral cavity primary tumors $(p=0.10)$ and were younger than patients without $P N I(p<0.01)$. Nine of 13 (69.3\%) oropharynx cancer patients with known HPV status had HPV-associated disease. Those with PNI were more likely to receive PORT: four of eight (50.0\%) with PNI versus six of 77 (7.8\%) without PNI $(\mathrm{p}<0.01)$. No patients received concurrent chemotherapy. Median follow-up was 46.4 months (95\% CI 7.885.3). 


\section{Cureus}

\begin{tabular}{|c|c|c|c|c|c|}
\hline & & Overall & PNI Negative & PNI Positive & P-value \\
\hline Total & & 85 & 77 & 8 & \\
\hline Age (median [range]) & & $66.0[20.0,97.0]$ & $66.0[20.0,97.0]$ & $53.0[36.0,68.0]$ & $<0.01$ \\
\hline \multirow[t]{2}{*}{$\operatorname{Sex}(\%)$} & Female & $32(37.6)$ & $29(37.7)$ & $3(37.5)$ & 1.00 \\
\hline & Male & $53(62.4)$ & $48(62.3)$ & $5(62.5)$ & \\
\hline \multirow[t]{3}{*}{ Site Category (\%) } & Oral Cavity & $53(62.4)$ & $45(58.4)$ & $8(100.0)$ & 0.10 \\
\hline & Oropharynx & $17(20.0)$ & $17(22.1)$ & $0(0.0)$ & \\
\hline & Larynx/Hypopharynx & $15(17.6)$ & $15(19.5)$ & $0(0.0)$ & \\
\hline \multirow[t]{12}{*}{ Tumor Site (\%) } & Base of Tongue & $6(7.1)$ & $6(7.9)$ & $0(0.0)$ & 0.05 \\
\hline & Buccal Mucosa & $5(6.0)$ & $5(6.6)$ & $0(0.0)$ & \\
\hline & Floor of Mouth & $5(6.0)$ & $4(5.3)$ & $1(12.5)$ & \\
\hline & Gingiva & $7(8.3)$ & $6(7.9)$ & 1 (12.5) & \\
\hline & Glottic Larynx & $6(7.1)$ & $6(7.9)$ & $0(0.0)$ & \\
\hline & Lip & $5(6.0)$ & $3(3.9)$ & $2(25.0)$ & \\
\hline & Posterior Pharyngeal Wall & $1(1.2)$ & $1(1.3)$ & $0(0.0)$ & \\
\hline & Retromolar Trigone & $1(1.2)$ & $0(0.0)$ & $1(12.5)$ & \\
\hline & Supraglottic Larynx & 7 (8.3) & 7 (9.2) & $0(0.0)$ & \\
\hline & Soft Palate & $1(1.2)$ & $1(1.3)$ & $0(0.0)$ & \\
\hline & Tongue & $30(35.7)$ & 27 (35.5) & $3(37.5)$ & \\
\hline & Tonsil & $10(11.9)$ & $10(13.2)$ & $0(0.0)$ & \\
\hline \multirow[t]{3}{*}{ Tobacco Use (\%) } & Current/Former & $56(65.9)$ & $48(62.3)$ & $8(100.0)$ & 0.10 \\
\hline & Never & $23(27.1)$ & $23(29.9)$ & $0(0.0)$ & \\
\hline & Smokeless Only & $6(7.1)$ & $6(7.8)$ & $0(0.0)$ & \\
\hline \multirow{2}{*}{ Pathologic T Stage (\%) } & $\mathrm{p} 11$ & $49(57.6)$ & 44 (57.1) & $5(62.5)$ & 1.00 \\
\hline & р12 & $36(42.4)$ & $33(42.9)$ & $3(37.5)$ & \\
\hline \multirow{2}{*}{ Pathologic N Stage (\%) } & pNO & $69(81.2)$ & $63(81.8)$ & $6(75.0)$ & 0.64 \\
\hline & pN1 & 16 (18.8) & 14 (18.2) & $2(25.0)$ & \\
\hline \multirow{2}{*}{ Neck Dissection (\%) } & No & $10(11.8)$ & $10(13.0)$ & $0(0.0)$ & 0.61 \\
\hline & Yes & $15(88.2)$ & $67(87.0)$ & $8(100.0)$ & \\
\hline \multirow{2}{*}{ Radiotherapy (\%) } & No & $15(88.2)$ & $71(92.2)$ & $4(50.0)$ & $<0.01$ \\
\hline & Yes & 10 (11.8) & $6(7.8)$ & $4(50.0)$ & \\
\hline \multirow{3}{*}{ Radiotherapy Neck Target (\%) } & Bilateral Neck & $5(50.0)$ & $3(50.0)$ & $2(50.0)$ & 0.33 \\
\hline & Primary Only & $3(30.0)$ & $1(16.7)$ & $2(50.0)$ & \\
\hline & Unknown & $2(20.0)$ & $2(33.3)$ & $0(0.0)$ & \\
\hline adıotherapy Dose (Gy & & $60.0[60$. & $60.0[60$. & 60.0 & $\mathrm{~N}$ \\
\hline
\end{tabular}

\section{TABLE 1: Patient Characteristics and Treatment by Perineural Invasion}

Gy: Gray; PNI: perineural invasion; NA: not applicable. All staging according to the American Joint Committee on Cancer 7th Edition. 


\section{Cureus}

In total, 17 patients experienced locoregional recurrence at a median time to recurrence of 9.2 months (95\% CI 5.0-27.7). Patterns of locoregional recurrence were as follows: local only $(n=6)$, regional only $(n=6)$, and local and regional $(n=5)$. Kaplan-Meier estimates of LRC at two and five years were 81.4\% (95\% CI 73.3-90.4) and 78.5\% (95\% CI 69.9-88.2), respectively (Figure 1). There were no differences in LRC between PNIpositive and PNI-negative groups ( $\mathrm{p}=0.73$ ). LRC was not impacted by PORT $(\mathrm{p}=0.39)$. Of the four patients with PNI as the sole pRF that did not receive PORT, none experienced locoregional failure. Due to the low number of PNI positive patients, comparisons within that group were not possible. Of the 17 patients that failed, 14 were salvaged with surgical resection alone $(n=8)$, surgery followed by PORT $(n=2)$, resection followed by PORT with concurrent chemotherapy $(n=2)$, or RT alone $(n=2)$.

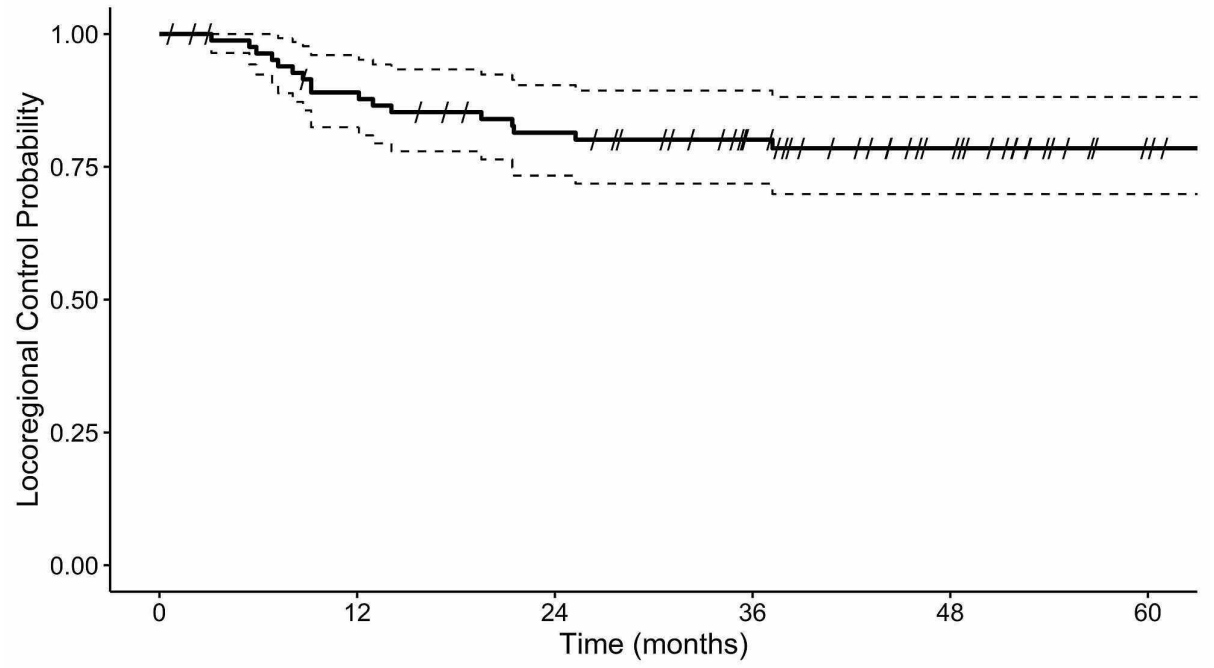

Number at risk

\begin{tabular}{|c|c|c|c|c|c|c|}
\hline All & 85 & 72 & 63 & 50 & 36 & 21 \\
\hline & 0 & 12 & 24 & 36 & 48 & 60 \\
\hline
\end{tabular}

FIGURE 1: Kaplan-Meier plot of locoregional control after primary surgical management of head and neck squamous cell cancer in pathologically low-risk patients irrespective of PNI status.

PNI: perineural invasion

Two- and five-year OS rates were 89.2\% (95\% CI 82.8-96.1) and 77.4\% (95\% CI 68.2-87.8), respectively. Corresponding estimates of DSS were 95.1\% (95\% CI 90.4-99.9) and 90.8\% (95\% CI 84.9-97.6), respectively (Figure 2). 


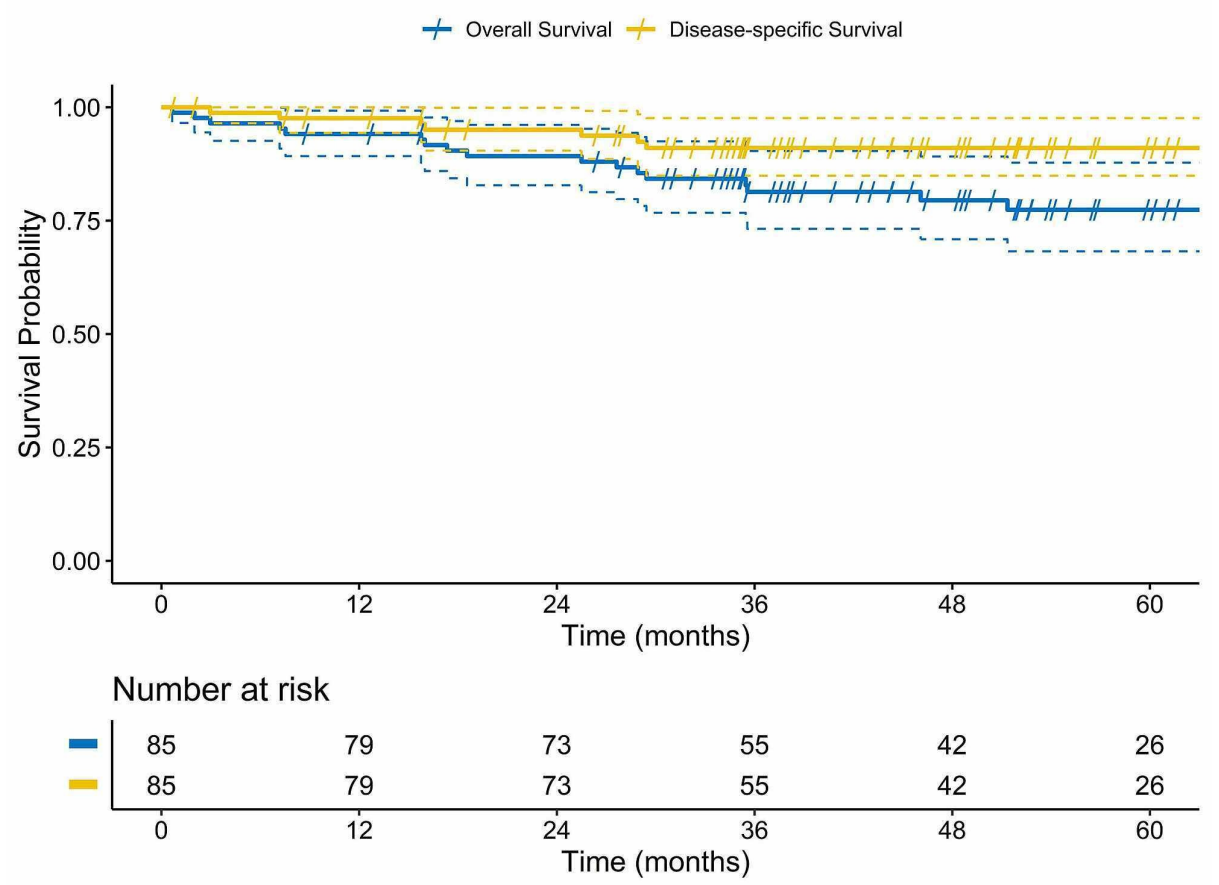

FIGURE 2: Kaplan-Meier plot of overall survival and disease-specific survival.

\section{Discussion}

Radiation therapy is routinely recommended for resected HNSCC with pRFs including pT3-4 disease, multiple lymph nodes, LVSI, PNI, close/positive margins, and ENE [14,15]. These risk factors frequently occur in combination and portend an increased risk of locoregional failure after surgery alone. In rare cases (less than $1 \%$ of patients treated at our institution), the tumor is positive for PNI as the sole pRF in the absence of other high-risk features. In these cases, the optimal postoperative approach is difficult to determine. Not surprisingly, PORT was given to half the patients with PNI as the sole risk factor. Notably, the LRC identified in our study is consistent with prior reports of low-risk patients $[4,9,10]$. However, the fact that four of eight patients did not receive PORT demonstrates the relevance of this issue.

A study evaluating the prognostic impact of PNI on patients with surgically resected oral cavity cancers with pathologically negative necks found that PNI was associated with worse LRC and disease-free interval [5]. However, the multivariable analyses identifying these associations included high grade and perivascular invasion, the frequency of which as they relate to PNI were not reported. Due to its frequently strong association with other pRFs, analyses including unselected patients with a variety of risk factors are limited in their ability to truly discriminate the impact of PNI itself $[8,16]$. Further large, multi-institutional studies are needed to assess the impact of PNI as the sole risk factor on patients with resected HNSCC.

A factor worth consideration is the extent and size of PNI in the tumor specimen. Large-nerve involvement and extensive PNI are frequently associated with disease recurrence in cutaneous HNSCC [17-19]. A pathologic risk assessment tool for oral cavity squamous cell carcinoma that included the size of nerves involved (in addition to lymphocytic tumoral infiltrate and worst pattern of invasion) has been associated with survival and disease-free survival $[4,20]$. Large nerve involvement was categorized as carcinoma tracking along or within a nerve with a diameter $\geqslant 1 \mathrm{~mm}$, while small nerve involvement included only nerves $<1 \mathrm{~mm}$ in diameter. The definition of extensive PNI is inconsistent in the literature but often is applied in the case of involvement of $\geqslant 2$ nerves [21]. Given the lack of clear evidence in the literature, adjuvant radiotherapy should remain a strong consideration, particularly in the presence of large nerve or multifocal PNI [12]. These considerations do not take into account clinical or radiographic perineural tumor spread from mucosal, salivary, or cutaneous malignancies, all of which warrant PORT [21]. This study specifically evaluates the impact of incidental pathologic PNI (without clinical findings suggestive of nerve involvement) on disease control.

This study is limited by its retrospective nature and the small sample size of pathologically low-risk patients with or without PNI. By limiting the analysis to pathologically low-risk patients with or without PNI and excluding other commonly accepted risk factors, we attempted to minimize any confounding factors and isolate PNI as the single pRF of interest. This limited our power to potentially identify differences between groups with regard to PNI and the use of PORT and introduces potential selection bias with regard to the 
utility of adjuvant therapy. Our institution's large overall head and neck patient volume allowed us to identify and describe a rare circumstance occurring in $<1 \%$ of patients undergoing upfront surgical resection.

\section{Conclusions}

PNI as the sole pRF after resection of HNSCC is an extremely rare circumstance. It is associated with younger age, oral cavity primary tumor, and the receipt of PORT. Since very few patients with PNI did not receive PORT, no conclusions can be made regarding its utility in this scenario, and it remains the standard of care. Further study is warranted, optimally including multiple institutions to pool data and improve statistical power.

\section{Additional Information \\ Disclosures}

Human subjects: Consent was obtained or waived by all participants in this study. Wake Forest University Health Sciences IRB issued approval IRB00047017. This study is approved by the Wake Forest University Health Sciences IRB. Animal subjects: All authors have confirmed that this study did not involve animal subjects or tissue. Conflicts of interest: In compliance with the ICMJE uniform disclosure form, all authors declare the following: Payment/services info: All authors have declared that no financial support was received from any organization for the submitted work. Financial relationships: All authors have declared that they have no financial relationships at present or within the previous three years with any organizations that might have an interest in the submitted work. Other relationships: All authors have declared that there are no other relationships or activities that could appear to have influenced the submitted work.

\section{References}

1. Batsakis JG: Nerves and neurotropic carcinomas. Ann Otol Rhinol Laryngol. 1985, 94:426-427.

2. Soo KC, Carter RL, O'Brien CJ, Barr L, Bliss JM, Shaw HJ: Prognostic implications of perineural spread in squamous carcinomas of the head and neck. Laryngoscope. 1986, 96:1145-1148. 10.1288/00005537198610000-00015

3. Fagan JJ, Collins B, Barnes L, D'Amico F, Myers EN, Johnson JT: Perineural invasion in squamous cell carcinoma of the head and neck. Arch Otolaryngol Head Neck Surg. 1998, 124:637-640. 10.1001/archotol.124.6.637

4. Brandwein-Gensler M, Teixeira MS, Lewis CM, et al.: Oral squamous cell carcinoma: histologic risk assessment, but not margin status, is strongly predictive of local disease-free and overall survival. Am J Surg Pathol. 2005, 29:167-178. 10.1097/01.pas.0000149687.90710.21

5. Chinn SB, Spector ME, Bellile EL, et al.: Impact of perineural invasion in the pathologically N0 neck in oral cavity squamous cell carcinoma. Otolaryngol Head Neck Surg. 2013, 149:893-899. $10.1177 / 0194599813506867$

6. Ballantyne AJ, McCarten AB, Ibanez ML: The extension of cancer of the head and neck through peripheral nerves. Am J Surg. 1963, 106:651-667. 10.1016/0002-9610(63)90074-6

7. Byers RM, O'Brien J, Waxler J: The therapeutic and prognostic implications of nerve invasion in cancer of the lower lip. Int J Radiat Oncol Biol Phys. 1978, 4:215-217. 10.1016/0360-3016(78)90140-2

8. Rahima B, Shingaki S, Nagata M, Saito C: Prognostic significance of perineural invasion in oral and oropharyngeal carcinoma. Oral Surg Oral Med Oral Pathol Oral Radiol Endod. 2004, 97:423-431. 10.1016/j.tripleo.2003.10.014

9. Peters LJ, Goepfert H, Ang KK, et al.: Evaluation of the dose for postoperative radiation therapy of head and neck cancer: first report of a prospective randomized trial. Int J Radiat Oncol Biol Phys. 1993, 26:3-11. 10.1016/0360-3016(93)90167-t

10. Ang KK, Trotti A, Brown BW, et al.: Randomized trial addressing risk features and time factors of surgery plus radiotherapy in advanced head-and-neck cancer. Int J Radiat Oncol Biol Phys. 2001, 51:571-578. 10.1016/s0360-3016(01)01690-x

11. Rosenthal DI, Mohamed ASR, Garden AS, et al.: Final report of a prospective randomized trial to evaluate the dose-response relationship for postoperative radiation therapy and pathologic risk groups in patients with head and neck cancer. Int J Radiat Oncol Biol Phys. 2017, 98:1002-1011. 10.1016/j.ijrobp.2017.02.218

12. Bur AM, Lin A, Weinstein GS: Adjuvant radiotherapy for early head and neck squamous cell carcinoma with perineural invasion: a systematic review. Head Neck. 2016, 38:2350-2357. 10.1002/hed.24295

13. Shim SJ, Cha J, Koom WS, Kim GE, Lee CG, Choi EC, Keum KC: Clinical outcomes for T1-2N0-1 oral tongue cancer patients underwent surgery with and without postoperative radiotherapy. Radiat Oncol. 2010, 5:43. 10.1186/1748-717X-5-43

14. National Comprehensive Cancer Network: Head and Neck Cancers (Version 2.2020) . (2020). Accessed: January 6, 2021: https://www.nccn.org/professionals/physician gls/pdf/head-and-neck.pdf.

15. Kiyota N, Tahara M, Fujii M: Adjuvant treatment for post-operative head and neck squamous cell carcinoma. Jpn J Clin Oncol. 2015, 45:2-6. 10.1093/ijco/hyu195

16. Lanzer M, Gander T, Kruse A, Luebbers HT, Reinisch S: Influence of histopathologic factors on pattern of metastasis in squamous cell carcinoma of the head and neck. Laryngoscope. 2014, 124:160-166. 10.1002/lary.24458

17. Carter JB, Johnson MM, Chua TL, Karia PS, Schmults CD: Outcomes of primary cutaneous squamous cell carcinoma with perineural invasion: an 11-year cohort study. JAMA Dermatol. 2013, 149:35-41. 10.1001/jamadermatol.2013.746 


\section{Cureus}

18. Balamucki CJ, Mancuso AA, Amdur RJ, et al.: Skin carcinoma of the head and neck with perineural invasion . Am J Otolaryngol. 2012, 33:447-454. 10.1016/j.amjoto.2011.11.004

19. Sapir E, Tolpadi A, McHugh J, et al.: Skin cancer of the head and neck with gross or microscopic perineural involvement: patterns of failure. Radiother Oncol. 2016, 120:81-86. 10.1016/j.radonc.2016.06.011

20. Brandwein-Gensler M, Smith RV, Wang B, et al.: Validation of the histologic risk model in a new cohort of patients with head and neck squamous cell carcinoma. Am J Surg Pathol. 2010, 34:676-688. 10.1097/PAS.0b013e3181d95c37

21. Bakst RL, Glastonbury CM, Parvathaneni U, Katabi N, Hu KS, Yom SS: Perineural invasion and perineural tumor spread in head and neck cancer. Int J Radiat Oncol Biol Phys. 2019, 103:1109-1124.

10.1016/j.ijrobp.2018.12.009 